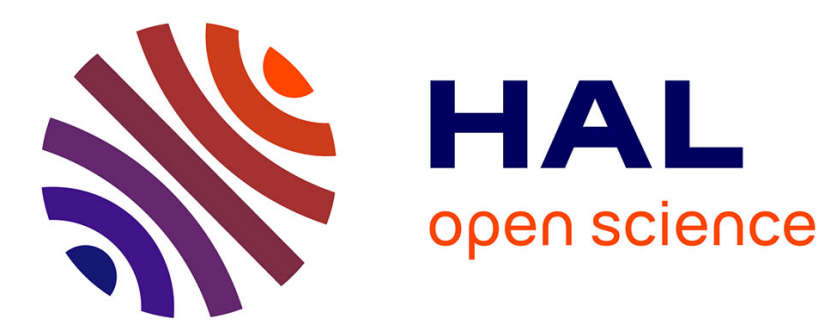

\title{
EIAH : Environnements Imaginaires pour l'Apprentissage Humain
}

Jacques Wallet

\section{To cite this version:}

Jacques Wallet. EIAH : Environnements Imaginaires pour l'Apprentissage Humain. STICEF (Sciences et Technologies de l'Information et de la Communication pour l'Éducation et la Formation), 2006, Forum de Discussion en Education, 13, pp.201-207. 10.3406/stice.2006.933 . hal-00697596

\section{HAL Id: hal-00697596 https://hal.science/hal-00697596}

Submitted on 15 May 2012

HAL is a multi-disciplinary open access archive for the deposit and dissemination of scientific research documents, whether they are published or not. The documents may come from teaching and research institutions in France or abroad, or from public or private research centers.
L'archive ouverte pluridisciplinaire HAL, est destinée au dépôt et à la diffusion de documents scientifiques de niveau recherche, publiés ou non, émanant des établissements d'enseignement et de recherche français ou étrangers, des laboratoires publics ou privés. 


\title{
EIAH : Environnements Imaginaires pour I'Apprentissage Humain
}

\author{
Jacques WALLET LUniversité de Rouen.
}

$\circ$

\section{Références}

Le présent texte propose une démarche peu habituelle en matière de technologies, fussent-elles pensées pour l'éducation: s'intéresser à de vieilles images. Des illustrations qui datent de la fin du XIXème siècle, une époque ou un saut technologique pour reprendre l'expression de A. T. Hall (Hall, 1996) lié aux progrès dans la communication déchaîne les imaginations : inventions du téléphone (1876), du phonographe (1878), tandis que l'invention ${ }^{\frac{1}{}}$ du cinématographe (1895) est pressentie

Entre anTICipation et «anTICipassion », pourrait-on dire, qui y-a-t-il de commun entre ces images troublantes et le débat contemporain sur les usages des technologies dans l'éducation et la formation ? Les passerelles sont multiples. Le texte se propose d'extrapoler à partir de ces « inventions » imaginaires ; de questionner notre présent à la lumière d'un passé qui n'exista point...

Albert Robida, illustrateur reconnu en son époque est aussi un auteur de science fiction comparé par certains à Jules Verne. Dans son œuvre, les techniques et les objets de communication occupent une place importante dans la société du futur. Ces objets, ici le phonographisme, sont toujours imaginés et illustrés dans un contexte sociétal. Parfois aussi avec une touche d'humour... ainsi, personne dans l'omnibus parisien Madeleine-Bastille n'écoute les nouvelles, sans que nous sachions ce que ce terme recouvre: informations quotidiennes ou courts récits littéraires?

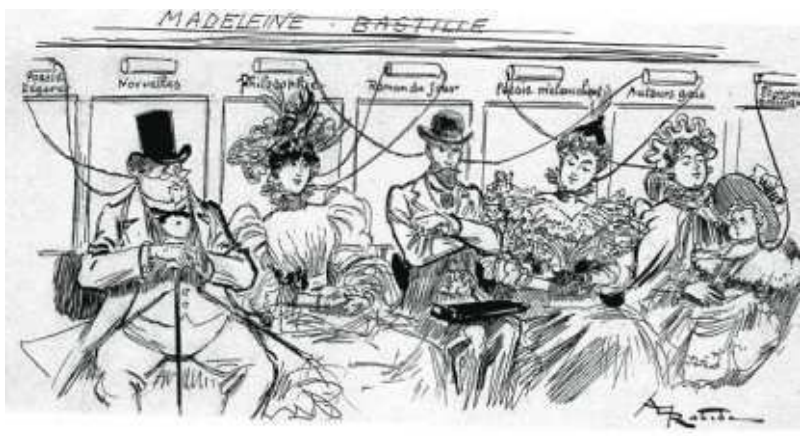

Albert Robida . «Le phonographisme futur s'offrira à nos petits fils dans toutes les circonstances de la vie »?

Les autres thèmes de ces «machines à diffuser» ont en revanche chacun leur auditeur. La galerie des personnages évoque le monde de la bourgeoisie, peut être en décalage avec les habituels passagers de la ligne de transport en commun. On remarque aussi que l'auteur n'a pas cherché ou n'a pas pensé à imaginer les habits du futur. La modélisation des apprenants, pour reprendre un concept et une terminologie contemporaine, très utilisé dans la communauté scientifique technophile, est cependant volontairement moquée. Le personnage central pratique le zapping entre «philosophie» et «auteurs gais » tandis que le poupard est attentif aux discours sur «l'économie politique »... Au delà, c'est bien le 
nomadisme des loisirs comme celui de l'apprentissage qui sont pressentis.

Sur une autre gravure de la même époque qui fit l'objet d'une publication antérieure (Wallet, 1997), c'est l'enseignement à distance médiatisé qui est pressenti. Il faut donner quelques éléments contextuels. A la rentrée des classes de l'automne 1880, le journal Le Charivari dénonce les ruses des jésuites pour continuer leur enseignement dans les établissements scolaires, cette fonction leur est interdite, après un décret pris en mars 1880, par le gouvernement de l'époque. Dans certains établissements scolaires, les jésuites dont «l'art de la dissimulation n'est plus à démontrer» sont accusés d'enseigner sous de fausses identités et certains religieux pousseraient même leurs élèves à émigrer avec eux à l'étranger... Une nouvelle « ruse » leur est prêtée par leurs adversaires : ceci explique le titre de la gravure.

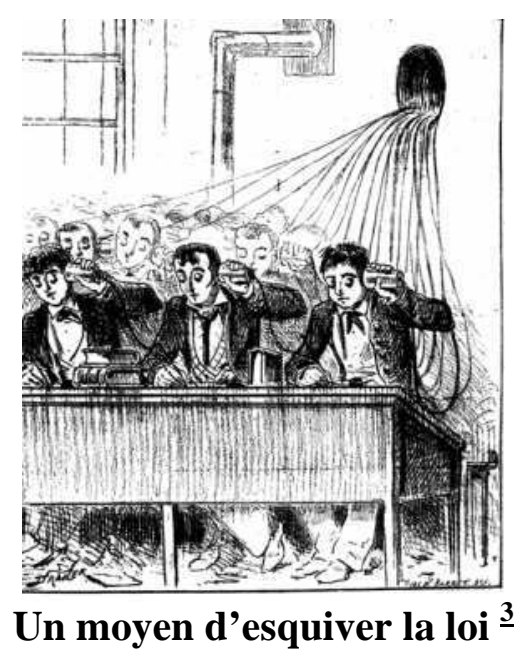

La gravure révèle sans doute en définitive, une double méfiance, un double appel implicite à la vigilance, contre les jésuites, certes, mais aussi contre le téléphone. L'usage de celui-ci pourrait se révéler: « un moyen d'esquiver la loi », tandis que la Société générale des téléphones poursuit dans Paris les travaux afin de raccorder ses premiers abonnés ${ }^{4}$. Le téléphone n'est pas encore rentré dans les pratiques sociales et encore moins dans les mœurs. Sa représentation sur la gravure le confirme. Elle peut laisser penser en effet que l'illustrateur n'a jamais vu de d'appareil téléphonique, il le caricature sous la forme d'une boîte de conserve qui évoque un jouet d'enfant, alors que les premiers postes commercialisés avaient une forme massive et cubique.

Pour qui aujourd'hui s'intéresse à la place des technologies dans l'éducation, le dispositif imaginé par l'illustrateur se charge d'une autre signification. Si l'on observe le sérieux et la concentration intellectuelle des collégiens, on peut supposer que l'illustrateur a sans doute sans le vouloir considéré comme résolue la difficulté principale pour l'organisation d'une formation à distance : celle de la distance pédagogique et psychologique des apprenants. Dans le cas présent, la motivation des uns et des autres semble extrême.

Dans notre lecture contemporaine et anachronique, forcément illégitime, le rôle de l'enseignant dans le dispositif envisagé est soulevé. Ici, la parole du maître est renforcée, sacralisée (ce qui est la moindre des choses pour celle d'un religieux) par la technologie. Cependant, aucun échange entre apprenants n'est perceptible sur la gravure. Au delà du cadre de l'image, y a t-il un comparse ou mieux un complice du jésuite expulsé : un tuteur ou un moniteur « hors champ » prêt à aider les élèves ? En tout cas, il leur ferait face car l'organisation pédagogique, avec des rangées de tables disposées de façon traditionnelle, en parallèle dans l'espace de la classe, reste classique. Enfin le dispositif, comme l'indique le titre de la gravure, est en marge de la loi. Le contrôler, le réglementer devient essentiel... et l'on songe alors aux problèmes juridiques contemporains du cyber-espace, particulièrement ceux rencontrés dans le $e$ learning... 
A la fin du XIXème siècle, le cross-média numérique est également anticipé. Pour utiliser une terminologie actuelle, la visioconférence, ici imaginée à des fins de communication inter-personnelles, est présente chez plusieurs illustrateurs ou auteurs, sujets de publications ou d'études récentes (Lange, 2001), (Delavaud, 2003).

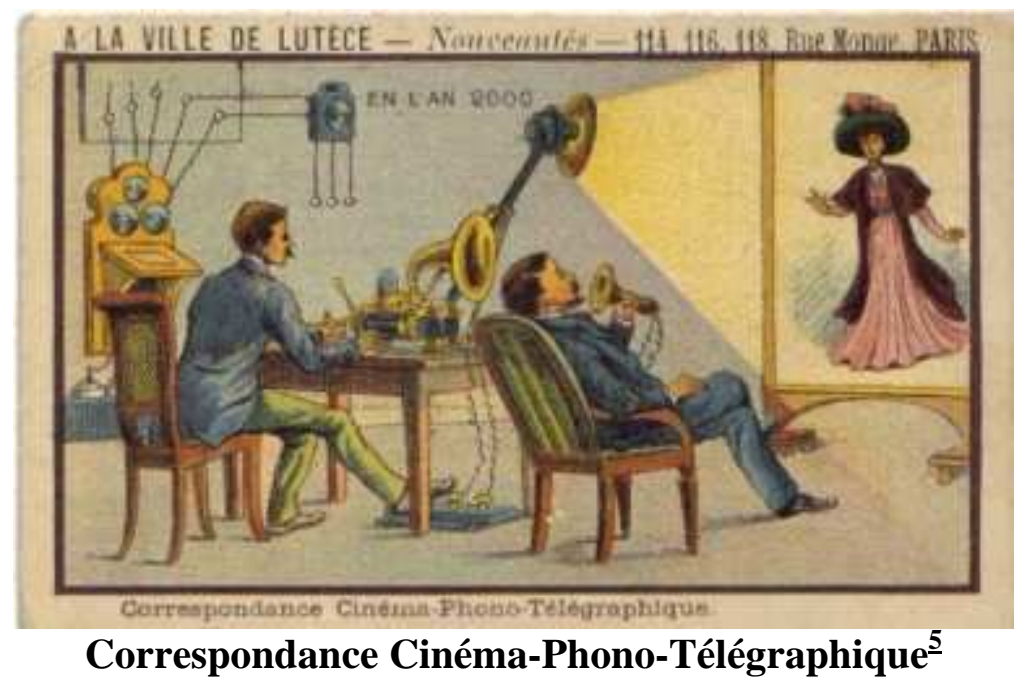

Mais parfois aussi, ces visioconférences ont explicitement un objectif éducatif.

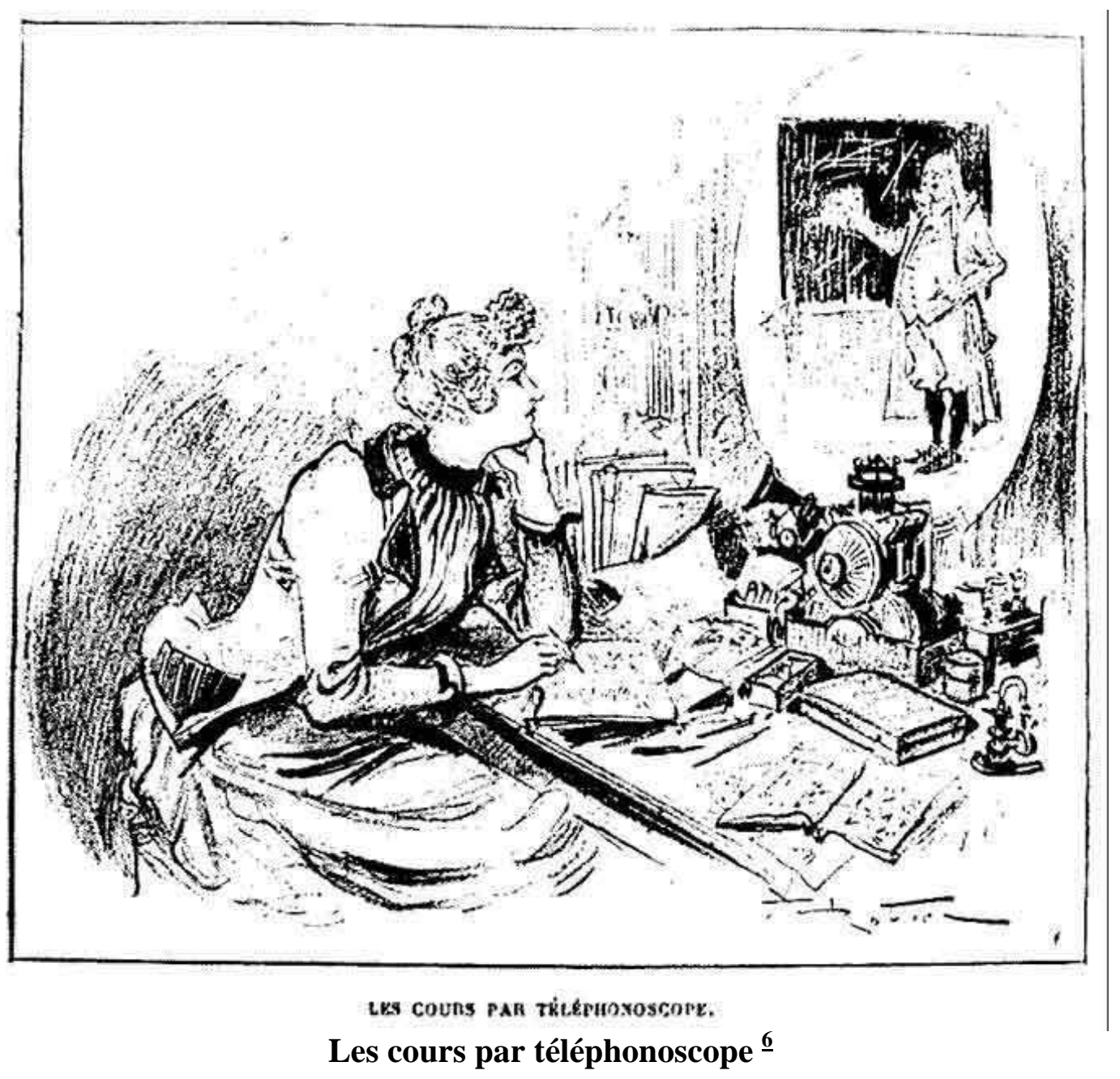


Pour l'auteur, la fonction ludique et culturelle de son imaginaire téléphonoscope, présent dans son roman illustré le vingtième siècle (1882) est première : faire venir le théâtre, l'opéra, la presse, la "visiophonie » à domicile. Il imagine déjà un modèle économique...

«Quand le téléphone fut universellement adopté, même pour les correspondants à grande distance, chacun s'abonna, moyennant un prix minime. Chaque maison eut son fil ramifié avec des bureaux de section, d'arrondissement et de région. De la sorte, pour une faible somme, on pouvait correspondre à tout heure, à n'importe quelle distance et sans dérangement, sans avoir à courir à un bureau quelconque. Le bureau de section établit la communication et tout est dit; on cause tant que l'on veut et comme on veut. Il y a loin, comme on voit, de là au tarif par mots de l'ancien télégraphe. ${\aleph^{\underline{7}}}^{-}$

Quelques années plus tard, dans un autre roman de science fiction, Le Vingtième Siècle, la vie électrique, dont l'action est censée se dérouler au milieu du vingtième siècle, le téléphonoscope (Robida utilise définitivement l'abréviation Télé ${ }^{\underline{x}}$... avec une majuscule !) a également une fonction éducative...

Ainsi héroïne Estelle Lacombe poursuit ses études depuis l'âge de douze ans ...

«sans quitter sa famille, uniquement par Télé. Précieux avantage pour, les familles éloignées de tout centre, qui ne sont plus forcées d'interner leurs enfants dans les lycées et collèges régionaux. Estelle avait fait toutes ses classes par Télé, sans sortir de chez elle...» $\underline{9}$

Notons au passage que la famille Lacombe a aussi recours au soutien scolaire à distance, puisque dans la continuité du texte, Robida précise :

«Elle suivait aussi de la même façon les cours de l'école centrale d'électricité de Paris et prenait, en outre, des répétitions par phonogrammes avec quelques maîtres renommés »

Timide, l'héroïne craque au moment des examens, elle l'avoue d'ailleurs à Georges $\frac{10}{}$ un autre personnage du roman rencontré par hasard sur Int... pardon sur Télé :

«faut-il vous l'avouer, j'ai déjà été retoquée deux fois...je pâlis sur mes cahiers, mais sans avancer beaucoup, il me semble... Hélas je ne mors pas facilement à cela... »

Car en effet, comme le précise Albert Robida, qui décidément a pensé à tout :

« elle n'avait pu passer ses examens par Télé, les règlements surannés s’y opposant, et, devant les maitres examinateurs, une timidité, qu'elle tenait un peu de son père, lui avait nui. »

Mais la véritable cause de l'échec d'Estelle est peut être à chercher en amont des épreuves d'examens... Sur la gravure, la relation pédagogique imaginée par Robida reste largement absconse et transmissive. Estelle semble rester dans l'expectative devant les démonstrations très théoriques de son professeur en redingote. Il y a là une autre leçon intemporelle à réaffirmer : le rapport entre innovation technologique et innovation pédagogique n'est pas intrinsèque... mais ceci est une autre histoire et cela devrait être une préoccupation bien contemporaine!

\section{Références}

\section{Références Bibliographiques}

DELAVAUD, G., (2003), coord. «Un siècle de télévision, anticipations, utopies, prospectives », revue les dossier de l'audiovisuel novembre/décembre, INA

HALL E-T. La dimension cachée - réédition , Points Seuils (essais) n89 - 1996

LANGE A. histoire de la télévision http://histv2.free.fr/ 
ROBIDA A., 1892 Le vingtième siècle, la vie électrique, Paris, Librairie illustrée, consultable en ligne sur : gallica, Bibliothèque Nationale http://visualiseur.bnf.fr/CadresFenetre?O=NUMM-101948\&M=notice\&Y=Image

WALLET, J., 1997, L'intuition de l'enseignement à distance sur une gravure ancienne, revue Sciences et techniques éducatives, éditions Hermès, volume 4, n³, p. 338-342

${ }^{1}$ Citer ces trois inventions, c'est ne pas en oublier d'autres qui jouèrent également un rôle décisif. Dater ces inventions ne signifie pas non plus méconnaître les polémiques autour de leurs paternités respectives.

$\underline{2}$ Dessin original, coll part. Voyages très extraordinaires dans le Paris d'Albert Robida, Paris, éditions Paris bibliothèque, 2005, p90

$\underline{3}$ Dessin de Draner et Barret, le Charivari, 12 octobre 1880, collection sur microfilm, Bibliothèque Nationale...»

4 Catherine Bertho, «La longue marche du téléphone », article dans la revue l’Histoire, Le Seuil, N67, mai 1984.

$\underline{5}$ Carte postale publicitaire pour un magasin parisien vers 1900 , « Raconte-moi la radio », site personnel de P. Dessapt. http://dspt.club.fr

$\underline{6}$ Albert Robida, La vie électrique le vingtième siècle, Paris, Librairie illustrée, 1892, page 25, bibliothèque Nationale

7 Albert Robida, Le vingtième siècle, 1882, cité par A. Lange

$\underline{8}$ Point de virus sur le Télé, mais il faut cependant utiliser des pantoufles isolatrices spéciales pour éviter tout risque d'électrocution

$\underline{9}$ Citations extraites de : Le vingtième siècle, la vie électrique (voir références bibliographiques)

10 Elle finira par épouser Georges, fils d'un grand savant, leur intrigue amoureuse somme toute très classique, permettra à Robida de décrire et d'illustrer tous les «progrès » de l'électricité (transports, médecine, culture, communication ...), mais aussi ses méfaits (guerre) ... les dernières lignes du livre qui évoquent les vacances des citadins sont ambiguës : "Nous allons recueillir des forces pour les luttes futures...continue à tourner avec les autres, ceux qui hélas! ne peuvent se donner ces quelques bonnes semaines de vacances, avec les malheureux ilotes trop profondément engagés dans les rudes engrenages, absorbante et terrifiante machine sociale !» La vie Electrique 1892.

\section{- A propos de l'auteur}

Jacques Wallet est professeur des universités en sciences de l'éducation à l'université de Rouen , laboratoire CIVIIC. Il travaille sur les usages des TICE, les dispositifs de formation à distance. Il est coordonnateur du réseau international res@tice de l'Agence Universitaire de la Francophonie et responsable pédagogique du campus numérique FORSE

Adresse : Université de Rouen, UFR de psychologie - département Sciences de l'Education, 1 rue Thomas Becket, 76821 Mont-Saint-Aignan cedex

Courriel : mailto:jacques.wallet@wanadoo.fr 
Revue sticef.org

Référence de l’article :

Jacques WALLET, EIAH : Environnements Imaginaires pour l'Apprentissage Humain, Revue STICEF, Volume 13, 2006, ISSN : 1764-7223, mis en ligne le 07/05/2006, http://sticef.org

(c) Revue Sciences et Technologies de 1'Information et de la Communication pour l’Éducation et la Formation, 2006 